\title{
Prospective evaluation of intense pulsed light and meibomian gland expression efficacy on relieving signs and symptoms of dry eye disease due to meibomian gland dysfunction
}

\author{
This article was published in the following Dove Press journal: \\ Clinical Ophthalmology \\ 2 May 2017 \\ Number of times this article has been viewed
}

\section{Steven J Dell' \\ Ronald N Gaster ${ }^{2}$ \\ Sheila C Barbarino' \\ Derek N Cunningham' \\ 'Dell Laser Consultants, Austin, TX, ${ }^{2}$ Gaster Eye Center, Beverly Hills, CA, USA}

Video abstract

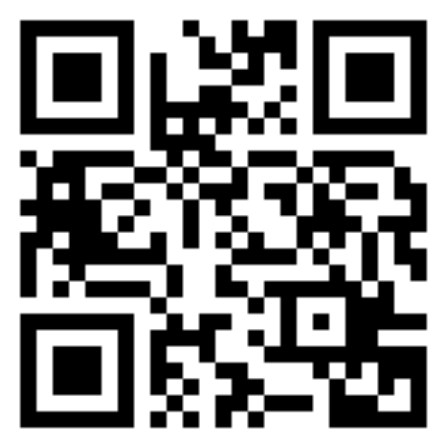

Point your SmartPhone at the code above. If you have a QR code reader the video abstract will appear. Or use:

http://youtu.be/01T6gmSn740
Correspondence: Steven J Dell

Dell Laser Consultants, 90 I South Mopac Expressway, Building 4, Suite 350, Austin, TX 78746, USA

Tel + I 5 I23470255

Email steven@dellmd.com
Purpose: The aim of this study was to estimate the efficacy of intense pulsed light (IPL), followed by meibomian gland expression (MGX), for reducing the number and severity of signs and symptoms of dry eye disease (DED) secondary to meibomian gland dysfunction (MGD).

Patients and methods: In a prospective study conducted in two sites, 40 subjects ( 80 eyes) with moderate to severe MGD were enrolled. Major inclusion criteria consisted of at least two of the following measures being compatible with DED in both eyes: tear breakup time (TBUT), meibomian gland score (MGS), corneal fluorescein staining (CFS), Standard Patient Evaluation of Eye Dryness (SPEED) questionnaire, and tear film osmolarity (TFO). Enrolled patients underwent four treatment sessions, 3 weeks apart. Each treatment included the administration of 10-15 pulses of IPL on the cheeks and nose, followed by MGX of the upper and lower eyelids. TBUT, MGS, CFS, SPEED, TFO, and lipid layer thickness (LLT) were measured at baseline (BL) and at 9, 12, and 15 weeks after BL.

Results: Due to different staining methods used for TBUT measurements, TBUT and CFS were analyzed separately for each site. From BL to the final follow-up, the number of signs compatible with DED decreased from $3.3 \pm 0.1$ to $1.4 \pm 0.1$. TBUT improved by $+93 \%(n=38$; $P<0.0001)$ and $+425 \%(\mathrm{n}=42 ; P<0.0001)$ for sites 1 and 2 , respectively. SPEED, MGS, and CFS improved by $-55 \%(\mathrm{n}=80 ; P<0.0001),-36 \%$ ( $\mathrm{n}=80 ; P<0.0001)$, and $-58 \%$ ( $\mathrm{n}=38 ; P<0.0001)$, respectively. In 20 eyes with abnormally elevated TFO at BL, TFO improved by $-7 \%(\mathrm{n}=20$; $P<0.005)$. LLT did not change ( $\mathrm{n}=38 ; P=0.88)$.

Conclusion: In subjects with moderate to severe MGD, IPL combined with MGX reduced the number and severity of symptoms and signs of DED. Except for LLT, all examined outcome measures significantly improved after 15 weeks. These results support the efficacy of IPL + MGX in relieving both signs and symptoms of DED secondary to MGD.

Keywords: dry eye, meibomian gland dysfunction, intense pulsed light

\section{Introduction}

Dry eye disease (DED) affects the quality of life of hundreds of millions of people around the globe. The most common form of DED is the evaporative form of the disease. ${ }^{1}$ By far, the most common cause of evaporative DED is meibomian gland dysfunction (MGD), with a prevalence of 5\%-20\% in western countries and $45 \%-70 \%$ in Asian populations. ${ }^{2}$ MGD is one of the most common disorders encountered by ophthalmologists. The pathogenesis of both conditions, MGD and DED, was recently described as two vicious cycles linked by inflammation: ${ }^{3}$ the MGD vicious 
cycle is triggered by various factors (eg, skin disorders, eyelid inflammation, and microbial infections), resulting in increased melting temperature of the meibum, blockage of the meibomian gland orifices, and, subsequently, inflammation and atrophy of the meibomian glands. As a result, tear film stability is compromised, exposing the cornea and triggering the DED vicious cycle, where inflammation of the ocular surface propagates to the lid margin and feeds back into the MGD cycle.

The current standard of care includes a variety of therapeutic strategies, including corticosteroids and other antiinflammatory drugs to control the inflammation, antibiotics to suppress bacterial infections, oral supplementations to change the composition of the meibum, warm compresses or thermal devices to soften the meibum, artificial tears and punctal plugs to keep the ocular surface moist and lid hygiene, blinking exercises, and mechanical expression of the meibomian glands. ${ }^{4}$ These therapies frequently provide only partial and temporary relief, perhaps because of our incomplete understanding of this complex pathology and perhaps because treatment has focused on addressing the symptoms, rather than the root cause.

Another approach is inspired by the well-known correlation between facial skin rosacea and MGD. A large proportion of patients with skin rosacea, $\sim 80 \%$, suffer also from ocular symptoms, the most prominent of them being MGD. ${ }^{5-8}$ Subjects with this skin disorder are three to four times more likely to suffer from symptomatic MGD. ${ }^{9}$ Given the correlation between facial skin rosacea and MGD, it seems plausible that treatment of skin rosacea might also improve MGD. Intense pulsed light (IPL) has demonstrated good clinical efficacy in skin rosacea. ${ }^{10,11}$ Could that type of treatment somehow benefit MGD as well? The first suggestion that IPL might improve MGD came from Toyos, who observed that rosacea subjects treated with IPL reported an improvement in their DED symptoms. ${ }^{12}$ Since then, several studies have shown that IPL therapy has a beneficial effect on MGD in patients with and without rosacea. ${ }^{13-17}$

In this prospective study, we present further evidence that IPL combined with meibomian gland expression (MGX) is effective in treating MGD. The IPL used in this study is based on Optima technology, which ensures uniform delivery of energy and therefore avoids under- or overtreatment.

\section{Patients and methods Patients}

Patients were recruited from, and treated at, two sites in the USA (site 1: Dell Laser Consultants, Austin, TX, USA; site 2: Gaster Eye Center, Beverly Hills, CA, USA).
General health and current/recent use of medications were screened to exclude patients for whom intense pulse light was contraindicated. Contact lens wear, recent ocular surgery, recent thermal treatment for DED (eg, LipiFlow), current use of punctal plugs, or recent expression of the meibomian glands also resulted in exclusion. Patients on standard of care such as warm compresses, lid hygiene, and artificial tears were allowed to continue these treatments.

Study enrollment consisted of consecutive patients who passed all exclusion criteria and satisfied the following inclusion criteria: able to read, understand, and sign an informed consent form; aged 18-80 years; Fitzpatrick skin type I-IV; at least five nonatrophied meibomian glands on each lower eyelid; and a current diagnosis of moderate to severe MGD in both eyes. This latter criterion was defined as two or more of the following conditions: 1) a tear breakup time $($ TBUT) $\leq 10 \mathrm{~s} ; 2$ ) a meibomian gland score (MGS; using the abbreviated MGD grading system for clinical trials) $>10$; 3) a corneal fluorescein staining (CFS; using the Baylor grading scheme) $\geq 10 ; 4$ ) a subjective symptom score (using the Standard Patient Evaluation of Eye Dryness [SPEED] questionnaire) $\geq 10$; and 5) a tear film osmolarity $(\mathrm{TFO}) \geq 310 \mathrm{mOsm} / \mathrm{L}$, or a TFO difference between the eye and its fellow eye $(\triangle T F O) \geq 8 \mathrm{mOsm} / \mathrm{L}$. The presence of skin rosacea was not a requirement for inclusion in the study.

This study adhered to the tenets of the Declaration of Helsinki, and the protocol was approved by an Institutional Review Board (Schulman, LUM-VBU-M22-15-01). All participants signed an informed consent form before enrollment.

\section{Study design}

This trial was conducted as a prospective, multisite, interventional, single-arm, exploratory, before-after study (NCT 02621593).

Enrolled patients underwent a series of four treatment sessions, 3 weeks apart. IPL was administered with the M22 Optima $^{\mathrm{TM}}$ IPL (Lumenis, Yokneam, Israel). Optima ${ }^{\mathrm{TM}}$ IPL technology ensures that fluence is constant and reproducible throughout each pulse, minimizing the risk of overtreatment (spikes) or undertreatment. To minimize the sensation of "snapping rubber band" that subjects treated with IPL occasionally feel, the treatment area was numbed with a topical anesthetic compound (eg, benzocaine 20\%-lidocaine 7\%-tetracaine 7\% compound gel). After protection of the eyes with disposable eye shields (Derm-Aid; Honeywell, Smithfield, RI, USA), Optima $^{\text {TM }}$ IPL was applied on a band of skin that extended from tragus to tragus (coronal axis) and on the cheeks from the maxillary process of the zygomatic bone up to the inferior orbital rim below the lower eyelids (longitudinal axis). Settings 
of the IPL, such as fluence and pulse interval, were dependent on skin type. Immediately after the IPL treatment, meibomian glands were expressed on both upper and lower eyelids of each eye. To minimize pain during this procedure, the eye was numbed with a solution of proparacaine $\mathrm{HCl} 0.5 \%$.

MGX was then performed by squeezing the meibomian glands with a meibomian gland expressor forceps, or with the aid of two Q-tips positioned on either sides of the meibomian glands.

Outcome measures were tested at the following four time points: on the same day and just before the first treatment session (hereafter referred to as the baseline [BL]); immediately before the fourth and final treatment sessions (first follow-up [FU1]); 3 weeks after the final treatment session (second follow-up [FU2]); and 6 weeks after the final treatment session (final follow-up [FU3]). From BL to the FU3, each patient was treated and followed up for a total of 15 weeks.

\section{Clinical tests}

At the BL and each of the three follow-ups, a series of clinical tests were performed to evaluate the TBUT (primary outcome measure) and secondary outcome measures, including subjective symptoms with the SPEED questionnaire, MGS, CFS, and TFO. Lipid layer thickness (LLT) was also measured in one of the sites, but it was not defined as a secondary outcome measure.

\section{TBUT}

After instillation of fluorescein on the ocular surface, the patient was asked to blink a few times to distribute the dye and then to close the eye once positioned at the slit lamp. A timer started when the patient opened his eye and stopped at the first sign of breakup. TBUT was evaluated as the average of three consecutive measurements. The following two sites used different methods of instillation: in site 1, the bulbar conjunctiva was touched with a Fluorescein Sodium Strip (Ful-Glo $0.6 \mathrm{mg}$; Akorn, Lake Forest, IL, USA); in site 2, a drop ( $\sim 50 \mu \mathrm{L})$ of fluorescein sodium and benonixate hydrochloride ophthalmic solution (0.25\%/0.4\%; Bausch \& Lomb, Rochester, NY, USA) was applied to the bulbar conjunctiva and any excess fluid was blotted off gently with a tissue.

TBUT $\leq 10 \mathrm{~s}$ is traditionally considered abnormal and consistent with DED. ${ }^{18}$ In this study, we used this criterion to distinguish between normal and abnormal TBUT values.

\section{Subjective symptoms}

Subjective symptoms were assessed with the validated SPEED questionnaire. ${ }^{19}$ The patient was asked to grade the severity and frequency of the following four symptoms, separately for each of his/her eyes: dryness/grittiness/scratchiness, soreness/irritation, burning/watering, and eye fatigue. For each of these symptoms, the patient scored the severity (from $0=$ no symptom to $4=$ intolerable) and the frequency (from $0=$ never to $3=$ constant $)$. SPEED was calculated as the sum of these eight subscores.

A SPEED value of $\geq 10$ is often considered consistent with moderate to severe DED symptoms. ${ }^{20}$ This is the cutoff value used in the study to distinguish between no/mild and moderate/severe dry eyes.

\section{MGS}

MGS was evaluated using the abbreviated MGD grading system for clinical trials. ${ }^{21}$ This compound score is a sum of subscores, including thickening of the upper lid margin (from $0=$ normal to $3=$ severe), vascularity of the upper lid margin (from $0=$ normal to $3=$ severe), telangiectasia of the upper lid margin (from $0=$ none to $3=$ more than 5 ), number of plugged glands of the 10 central glands in the upper eyelid, quality of the meibum (from $0=$ clear to $3=$ solid), expressibility (from $0=$ minimal pressure to $3=$ heavy pressure), and gland dropout from the central two-thirds of the lower eyelid. In our study, we used a score of $>10$ for categorizing DED as moderate to severe. ${ }^{21}$

\section{CFS}

Following instillation of fluorescein on the ocular surface, the cornea was examined under blue light illumination and a yellow filter. CFS was estimated using the Baylor grading scheme: ${ }^{22}$ staining of each of five zones of the cornea (central, temporal, nasal, superior, and inferior) was scored using the following 5-point scale: 0 dots $=0,1-5$ dots $=1,6-15$ dots $=2$, $16-30$ dots $=3$, and $>30$ dots $=4$. One point was added if there was a single area of confluent staining. Two points were added if there were at least two areas of confluent staining. According to Fenner and Tong, ${ }^{23}$ in evaporative dry eye, the mean Baylor score of each corneal zone ranges between 1.0 and 2.5. In our study, we used cutoff $>10$ for categorizing DED as moderate to severe.

\section{TFO}

TFO was evaluated by measuring the electrical impedance of a $50 \mathrm{~nL}$ sample collected from the lower meniscus, using an osmolarity measurement device (TearLan, San Diego, CA, USA). Different studies use slightly different cutoff values to distinguish between normal TFO and TFO consistent with DED, depending on the severity of dry eye and the preferred trade-off between sensitivity and specificity. ${ }^{24,25}$ The accepted values range from 305 to $318 \mathrm{mOsm} / \mathrm{L}$. 
In our study, we used a cutoff value of $310 \mathrm{mOsm} / \mathrm{L}$. In addition, an intereye difference of $\geq 8 \mathrm{mOsm} / \mathrm{L}$ is also considered as a characteristic signature of DED. ${ }^{24}$

\section{LLT}

LLT was measured using the LipiView interferometer (TearScience, Morrisville, NC, USA). LLT measurements were conducted in site 1 only, since site 2 did not have the device. The accepted cutoff values range between 60 and $75 \mathrm{~nm} .{ }^{26}$ In this study, we used a cutoff value of $60 \mathrm{~nm}$.

\section{Statistical analysis}

All statistical analyses were done using JMP 12.2.0 (SAS statistical software). Only eyes that completed all follow-ups were taken for the analysis. In 12 of 320 osmolarity measurements, values were missing due to malfunction of the device or other technical issues. In these cases, values were completed using a "last observation carried forward" strategy.

Descriptive statistics included proportions for categorical variables, mean \pm standard deviation $(S D)$ or mean \pm standard error of the mean (SEM) for continuous variables. Continuous variables were tested for normality with the Shapiro-Wilk test. Paired analysis allowed comparison of data before treatment and at each of the individual follow-ups. Except where mentioned, all paired analyses assumed nonparametric distributions and were performed with Wilcoxon signed rank test. Paired analyses for parametric distributions were done with two-tail paired $t$-test assuming equal variance. Longitudinal analysis was performed using a within-subjects multivariate analysis of variance ( $F$-test).

For categorical analysis, outcome measures were dichotomized according to the cutoff and conditions listed earlier. Odds ratios (ORs) were calculated using logistic regression. Differences were defined as statistically significant at the $\alpha=0.05$ level.

\section{Results}

\section{Flow}

Between September 2015 and July 2016, 46 patients were enrolled from two sites (site 1: 24 patients; site 2: 22 patients). One patient in site 2 was withdrawn after receiving a first treatment, due to the history of migraines that was not identified at screening. One patient in site 1 missed FU3 and is considered lost to follow-up. At the time of writing, four patients were still in various stages of the study and were not included in the analysis. Forty patients (80 eyes) completed all treatments and follow-up sessions and formed the statistical database for this article. Figure 1 is a flowchart that summarizes progress through the various phases of the
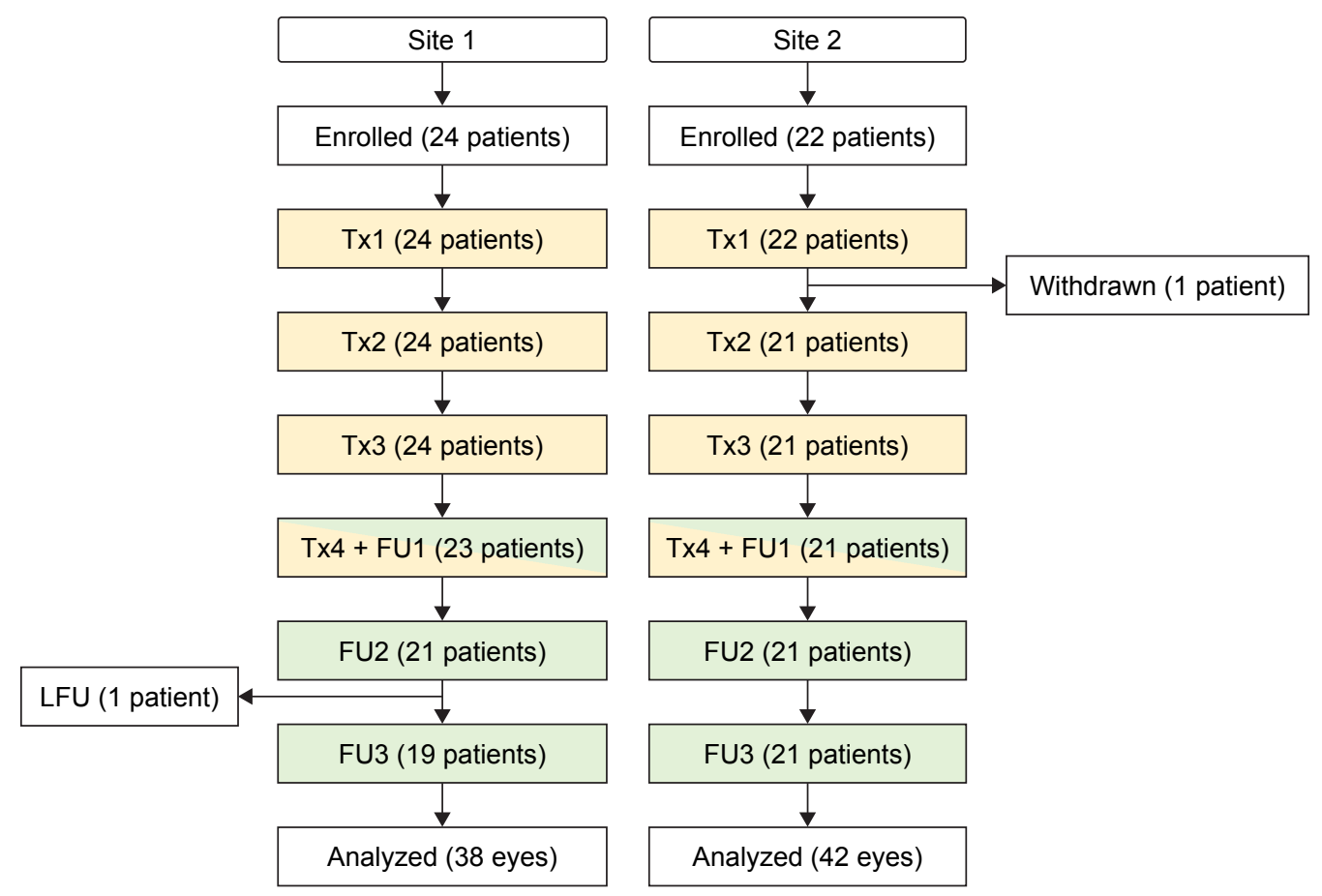

Figure I Study flow diagram.

Notes: Withdrawn case in site 2 received one IPL treatment but was withdrawn because it was discovered, after the treatment, that the patient has a history of migraines. Abbreviations: FU, follow-up; LFU, lost to follow-up; Tx, treatment session. 
Table I Demographic information

\begin{tabular}{|c|c|c|c|c|c|}
\hline & $\mathbf{N}$ & Age $(\mu \pm$ SD $)$ & Gender (male) & $\begin{array}{l}\text { Skin type } \\
\text { (Fitzpatrick scale) }\end{array}$ & $\begin{array}{l}\text { Baseline rosacea } \\
(0=\text { none; } 3=\text { severe })\end{array}$ \\
\hline Site I & 19 & $53.6 \pm 15.7$ & $58 \%$ & $2.8 \pm 0.5$ & $1.36 \pm 0.67$ \\
\hline Site 2 & 21 & $61.0 \pm 13.9$ & $43 \%$ & $2.6 \pm 0.7$ & $0.57 \pm 0.67$ \\
\hline Sites $1+2$ & 40 & $57.5 \pm 15.1$ & $50 \%$ & $2.7 \pm 0.6$ & $0.95 \pm 0.78$ \\
\hline Site I vs site 2 ( $P$-value) & & 0.08 & 0.41 & 0.38 & $<0.0001$ \\
\hline
\end{tabular}

Notes: Site I vs Site 2 were compared with two-tail $t$-test. A P-value less than the $\alpha$ level $(0.05)$ implies that the two sites are different. Abbreviation: SD, standard deviation.

study (enrollment, withdrawal, treatments, follow-ups, lost to follow-ups, and analysis).

\section{Demographics}

Patients in the two sites had similar characteristics, except for BL rosacea, which was on average slightly more severe in site 1 than in site 2 (Table 1). The mean age was $57.5 \pm 15.1$ years $(\mathrm{SD})$ and ranged between 23 and 77 years. More than $90 \%$ of the patients had a Fitzpatrick skin type of II or III. The vast majority of the patients (90\%) were Caucasian, with the rest distributed among Hispanic, Asian, or other minorities. Most patients $(70 \%)$ had no or mild signs of ocular rosacea at BL. Severity of skin rosacea at BL was distributed as follows: 12 (30\%), 19 (48\%), 8 (20\%), and 1 $(3 \%)$ had no rosacea, mild rosacea, moderate rosacea, and severe rosacea, respectively.

Previous management of DED included none (52.5\%), punctal plugs $(27.5 \%)$, LipiFlow (3\%), artificial tears (17.5\%), Restasis (10\%), and warm compresses (3\%). Some of the patients were managed with more than one method of treatment.

\section{TBUT (primary outcome measure)}

Because of the different staining methods used for TBUT measurements in the two sites, TBUT results were analyzed separately for each of the two sites (Figure 2). The difference between the two sites is discussed in the "Discussion" section.

In both sites, the average TBUT gradually increased from BL to the FU3, improving by $+93 \%$ in site $1(P<0.0001)$ and by $+425 \%$ in site $2(P<0.0001)$ (Table 2$)$. The proportion of eyes with normal TBUT values ( $>10 \mathrm{~s})$ also increased in both sites, from $2.6 \%$ to $53 \%$ in site 1 and from $36 \%$ to $100 \%$ in site 2 (Table 3 ).

In site 1 , the OR indicates that a treated eye was 41 times more likely to end up with a normal TBUT value (TBUT $>10 \mathrm{~s}$ ) at the FU3 than at the BL (Figure 3). OR was even larger when adjusted for age $(\mathrm{OR}=51)$. In site 2 , ORs could not be calculated for the FU3, because at this time point, all eyes had a TBUT value above the cutoff.

In a set of subgroup analyses performed for data collected in site 1 , the effects of age, BL rosacea, BL TBUT, and gender on TBUT were examined (Figure 4). Thirty-eight eyes were divided to subgroups according to age group (young vs old, using the median age of 58 years as cutoff), the severity of BL rosacea (none/mild vs moderate/severe), BL TBUT (using the median TBUT of $6 \mathrm{~s}$ as cutoff), and gender. All subgroups reached similar TBUT values at the FU3 (young vs old: $P=0.31$; none/mild vs moderate/severe $\mathrm{BL}$ rosacea: $P=0.57$; low BL TBUT $[\leq 6 \mathrm{~s}$ ] vs high BL TBUT [ $>6 \mathrm{~s}]$ : $P=0.91$; male vs female: $P=0.69$ ).
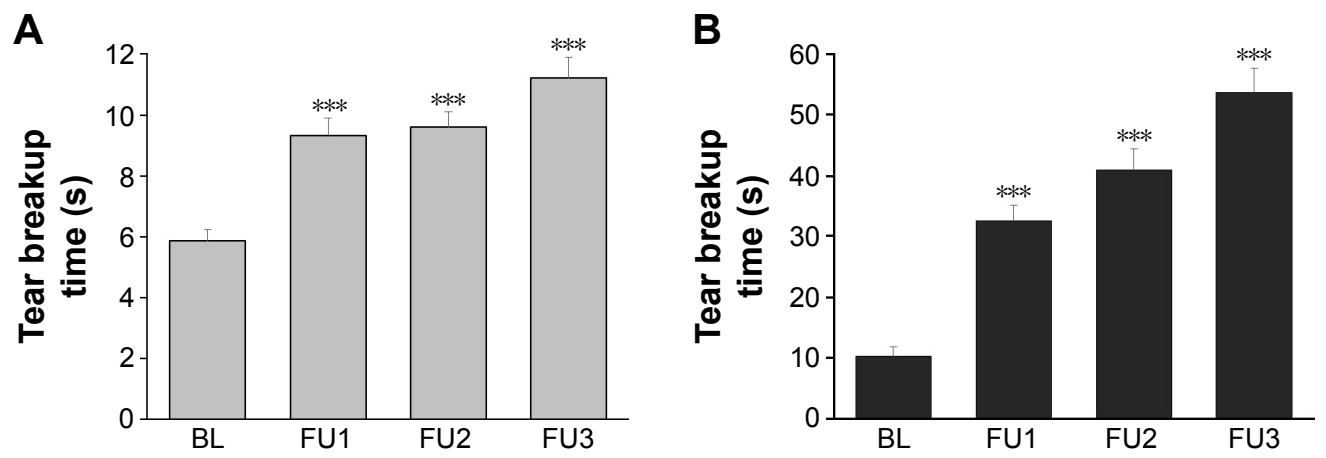

Figure $\mathbf{2}$ Longitudinal analysis of tear breakup time.

Notes: Error bars: standard error of the mean values. ***Wilcoxon signed rank test against $B L(P<0.00 I)$. (A) Site I and (B) site 2 .

Abbreviations: BL, baseline; FU, follow-up. 
Table 2 Numerical analysis

\begin{tabular}{|c|c|c|c|c|c|c|c|c|c|c|c|c|c|}
\hline \multirow{2}{*}{$\begin{array}{l}\text { Outcome } \\
\text { measure }\end{array}$} & \multirow[t]{2}{*}{ Sites } & \multirow[t]{2}{*}{$\mathbf{N}$} & \multirow{2}{*}{$\begin{array}{l}\text { BL } \\
\text { Mean } \\
\text { (SD) }\end{array}$} & \multicolumn{3}{|l|}{ FUI } & \multicolumn{3}{|l|}{ FU2 } & \multicolumn{3}{|l|}{ FU3 } & \multirow[t]{2}{*}{$\boldsymbol{P}_{\text {within_pt }}$} \\
\hline & & & & $\begin{array}{l}\text { Mean } \\
(\mathrm{SD})\end{array}$ & $\begin{array}{l}\text { Change } \\
\text { vs BL (\%) }\end{array}$ & $P$ & $\begin{array}{l}\text { Mean } \\
(\mathrm{SD})\end{array}$ & $\begin{array}{l}\text { Change } \\
\text { vs BL (\%) }\end{array}$ & $P$ & $\begin{array}{l}\text { Mean } \\
(S D)\end{array}$ & $\begin{array}{l}\text { Change } \\
\text { vs BL (\%) }\end{array}$ & $P$ & \\
\hline \multirow[t]{2}{*}{ TBUT (s) } & 1 & 38 & $5.8(2.3)$ & $9.3(3.5)$ & 60 & $<0.0001$ & $9.6(3.2)$ & 66 & $<0.0001$ & I I.2 (4.2) & 93 & $<0.0001$ & $<0.000$ I \\
\hline & 2 & 42 & $10.2(10)$ & 32 (I7) & 218 & $<0.0001$ & $4 I(22)$ & 300 & $<0.0001$ & $54(26)$ & 425 & $<0.0001$ & $<0.0001$ \\
\hline SPEED & $\mathrm{I}+2$ & 80 & $12.9(4.9)$ & $6.6(4.4)$ & -49 & $<0.005$ & $6.3(4.3)$ & -51 & $<0.005$ & $5.8(4.2)$ & -55 & $<0.001$ & $<0.0001$ \\
\hline MGS & $1+2$ & 80 & $20.3(7.2)$ & I3.4 (5.5) & -34 & $<0.01$ & $12.4(5.2)$ & -39 & $<0.01$ & $12.9(6.0)$ & -36 & $<0.05$ & $<0.000$ I \\
\hline \multirow[t]{2}{*}{ CFS } & 1 & 38 & $7.8(4.6)$ & $3.3(2.9)$ & -57 & $<0.0001$ & $4.5(3.5)$ & -43 & $<0.0001$ & $3.3(3.3)$ & -58 & $<0.0001$ & $<0.0001$ \\
\hline & 2 & 42 & $0.2(0.9)$ & I.0 (4.7) & 340 & 0.32 & $0.1(0.4)$ & -50 & 0.74 & $0.5(0.2)$ & 100 & 0.35 & 0.07 \\
\hline $\begin{array}{l}\text { TFO } \\
\text { (mOsm/L) }\end{array}$ & $1+2$ & 20 & $322.2(19)$ & $298.5(I 2)$ & -7 & $<0.0001$ & 297.9 (II) & -7.5 & $<0.0001$ & 297.8 (9) & -8 & $<0.0001$ & $<0.005$ \\
\hline $\begin{array}{l}\Delta \mathrm{TFO} \\
(\mathrm{mOsm} / \mathrm{L})\end{array}$ & $I+2$ & 24 & 17.8 (19) & $8.6(5.4)$ & -52 & $<0.0001$ & $8.8(6.9)$ & -51 & $<0.005$ & $6.3(4.2)$ & -65 & $<0.000 \mathrm{I}$ & 0.06 \\
\hline LLT (nm) & 1 & 38 & 79.3 (19) & $80.7(20)$ & 1.8 & 0.89 & 81.1 (22) & 2.3 & 0.73 & $79.4(21)$ & 0.2 & 0.93 & 0.88 \\
\hline
\end{tabular}

Notes: Change vs BL was calculated by subtracting the BL from the FU means and dividing by the BL mean. $P=$ probability that FU and BL are similar (two-tail Wilcoxon signed rank test): $P<0.05$ suggests that the distributions are different. $P_{\text {within_pt }}=$ probability that there was no change within subjects (repeated measures, MANOVA test). TBUT and CFS were analyzed per each site separately. TFO was analyzed only for eyes with an abnormally elevated TFO value ( $\geq 3 / 0 \mathrm{mOsm} / \mathrm{L})$ at BL. $\triangle T F O$, the difference of osmolarity between both eyes of a patient, was analyzed for patients with an abnormally elevated $\Delta \mathrm{TFO}(\geq 8 \mathrm{mOsm} / \mathrm{L})$ at $\mathrm{BL}$.

Abbreviations: BL, baseline; CFS, corneal fluorescein staining; FU, follow-up; LLT, lipid layer thickness; MANOVA, multivariate analysis of variance; MGS, meibomian gland score; SD, standard deviation; SPEED, Standard Patient Evaluation of Eye Dryness; TBUT, tear breakup time; TFO, tear film osmolarity.

Table 3 Categorical analysis

\begin{tabular}{|c|c|c|c|c|c|c|c|}
\hline Outcome measure & Normality criterion & Sites & $\mathbf{N}$ & BL, n (\%) & FUI, n (\%) & FU2, n (\%) & FU3, n (\%) \\
\hline \multirow[t]{2}{*}{ TBUT (s) } & $>10$ & I & 38 & I (2.6) & II (29) & $15(39)$ & $20(53)$ \\
\hline & & 2 & 42 & $15(36)$ & $39(93)$ & $40(95)$ & $42(100)$ \\
\hline SPEED & $<10$ & $\mathrm{I}+2$ & 80 & $18(22)$ & $65(81)$ & $61(76)$ & $67(84)$ \\
\hline MGS & $\leq 10$ & $1+2$ & 80 & $7(8.8)$ & $24(30)$ & $31(39)$ & $28(35)$ \\
\hline \multirow[t]{2}{*}{ CFS } & $<10$ & 1 & 38 & $24(63)$ & $38(100)$ & $34(89)$ & $36(95)$ \\
\hline & & 2 & 42 & $42(100)$ & $40(95)$ & $42(100)$ & $42(100)$ \\
\hline TFO (mOsm/L) & $<310$ & $\mathrm{I}+2$ & 20 & $0(0)$ & $17(85)$ & $17(85)$ & $17(85)$ \\
\hline$\Delta \mathrm{TFO}(\mathrm{mOsm} / \mathrm{L})$ & $<8$ & $1+2$ & 24 & $0(0)$ & $13(54)$ & $14(58)$ & $16(67)$ \\
\hline $\operatorname{LLT}(\mathrm{nm})$ & $<60$ & 1 & 38 & $29(76)$ & $31(82)$ & $31(76)$ & $28(74)$ \\
\hline
\end{tabular}

Notes: In this analysis, outcome measures were dichotomized to "normal" or "consistent with DED", according to the criteria listed under the column "Normality criterion". n, number of eyes with normal values.

Abbreviations: BL, baseline; CFS, corneal fluorescein staining; DED, dry eye disease; FU, follow-up; LLT, lipid layer thickness; MGS, meibomian gland score; SPEED, Standard Patient Evaluation of Eye Dryness; TBUT, tear breakup time; TFO, tear film osmolarity.

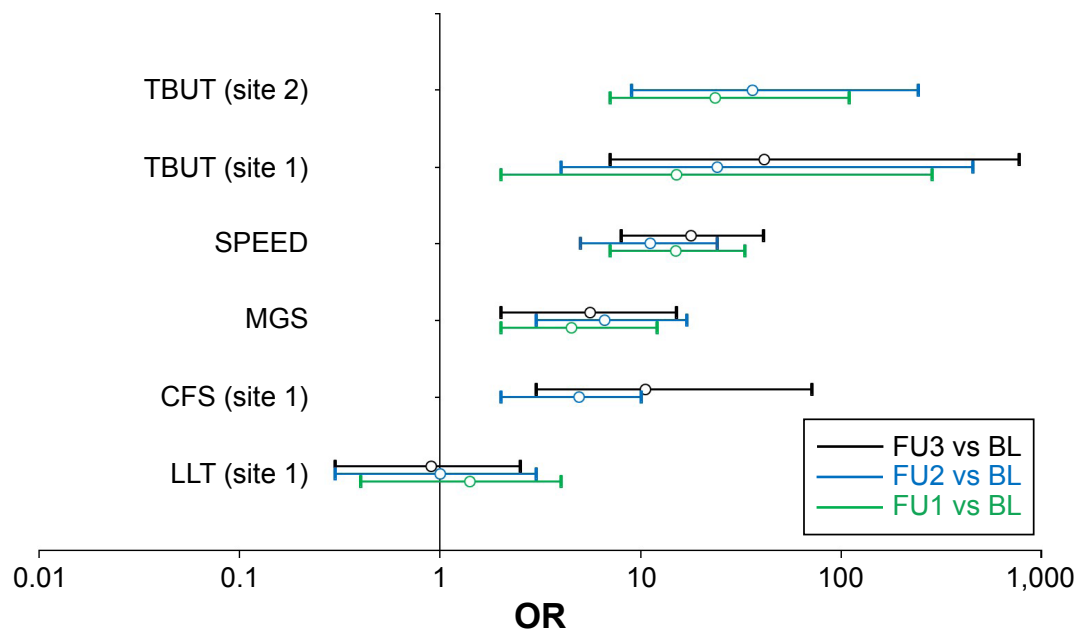

Figure 3 Forest plot of odds ratios for study measures.

Notes: Outcome measures were dichotomized as detailed in Table 3. Open circles and bars represent ORs and $95 \%$ confidence intervals, respectively. Green, blue, and black symbols show FUI vs BL, FU2 vs BL, and FU3 vs BL, respectively. ORs for which the $95 \%$ confidence interval do not cross OR $=\mathrm{I}$ are statistically significant. Undefined ORs are missing from this plot.

Abbreviations: BL, baseline; CFS, corneal fluorescein staining; FU, follow-up; LLT, lipid layer thickness; MGS, meibomian gland score; ORs, odds ratios; SPEED, Standard Patient Evaluation of Eye Dryness; TBUT, tear breakup time. 

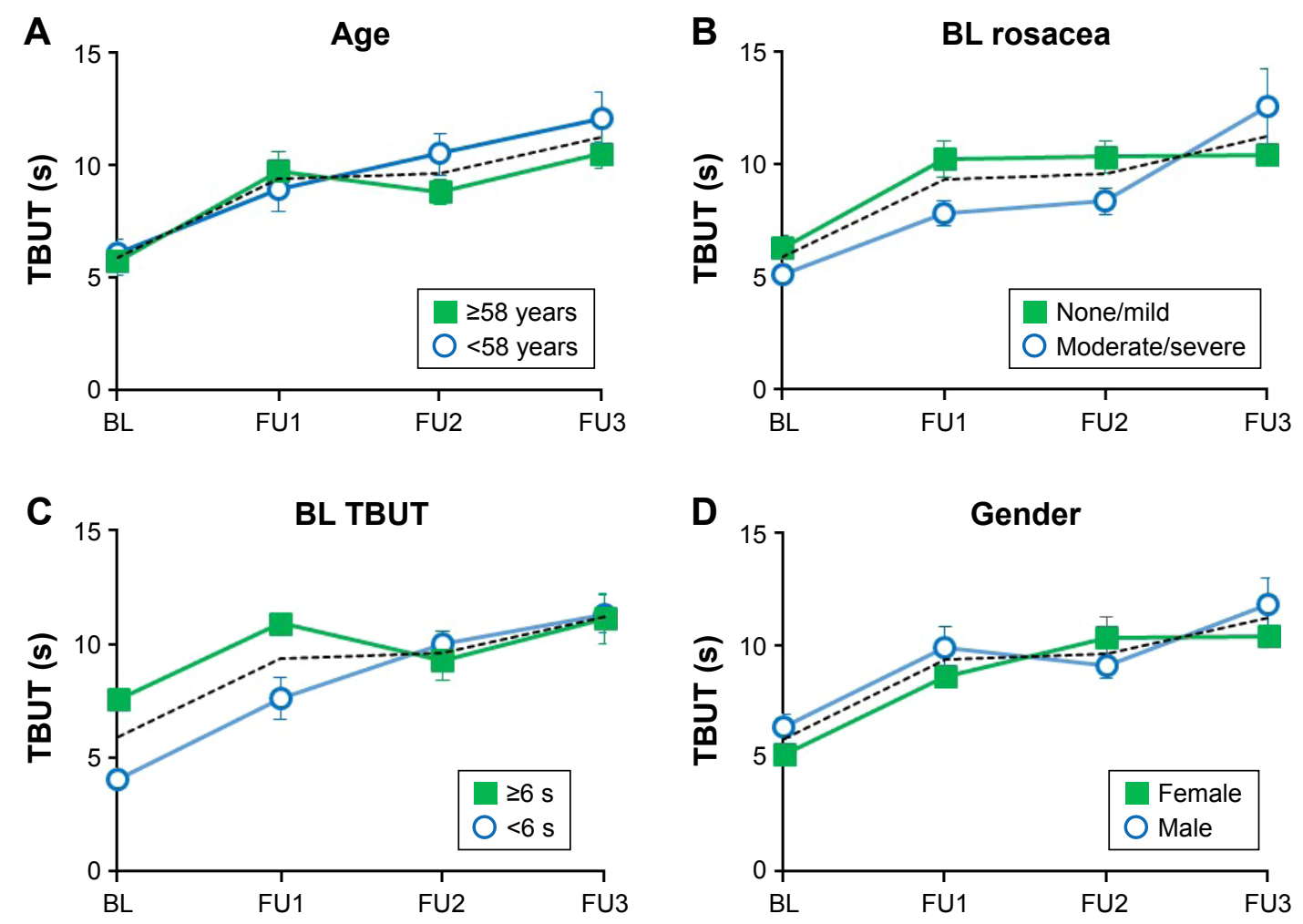

Figure 4 Subgroup analysis of TBUT (site I).

Notes: In each panel, the dotted line shows the TBUT data for the complete cohort (38 eyes). (A) Effect of age. Solid squares: patients aged $<58$ years ( $\mathrm{n}=18$ ); open circles: patients aged $>58$ years $(n=20)$. (B) Effect of baseline skin rosacea. Solid squares: patients with no or mild skin rosacea at $B L(n=24)$; open circles: patients with moderate or severe skin rosacea at BL $(n=14)$. (C) Effect of BL TBUT. Solid squares: eyes with TBUT $\geq 6 \mathrm{~s}(n=20)$; open circles: eyes with TBUT $<6 \mathrm{~s}(\mathrm{n}=18)$. (D) Effect of gender. Solid squares: females $(n=16)$; open circles: males $(n=22)$.

Abbreviations: BL, baseline; FU, follow-up; TBUT, tear breakup time.

\section{Subjective symptoms (SPEED)}

Subjective symptoms were evaluated using the SPEED questionnaires, performed for each eye separately. From BL to each of the three successive follow-ups, the average SPEED score decreased by $-49 \%,-51 \%$, and $-55 \%(P<0.0001)$ (Table 2). The proportion of eyes with normal SPEED values $(<10)$ increased from $22 \%$ at the BL to $84 \%$ at the FU3 (Table 3). ORs indicate that a normal SPEED value was 18 times more likely at the FU3 than at the BL (Figure 3).

All eight individual components of the SPEED score, including the severity and frequency of each of the four tested symptoms (dryness/scratchiness, soreness/irritation, burning/watering, and eye fatigue), followed similar changes in time (not shown).

\section{MGS}

The MGS is a compound score of the components outlined below. The overall MGS decreased from BL by $-34 \%,-39 \%$, and $-36 \%$ at the FU1, FU2, and FU3, respectively (Table 2). The proportion of eyes with normal $(<10)$ MGS quadrupled, from $9 \%$ at BL to $35 \%$ at the FU3 (Table 3). The ORs indicate that, compared to the BL, a normal MGS was 5.6 times more likely at the FU3, compared to prior to treatment (Figure 3). All nine individual components of MGS improved from BL to the FU3.

In the upper lid margin, the proportion of eyes with no telangiectasia doubled (from $31 \%$ to $64 \%$ ) and the average number of telangiectasia decreased by $65 \%(P<0.0001)$; the proportion of eyes with no vascularity increased from $15 \%$ to $25 \%$, and the average change was from mild/moderate to mild $(-29 \%, P<0.0001)$; and the proportion of eyes with no thickening increased from $7.5 \%$ to $14 \%$, while the average thickening decreased by $16 \%(P<0.01)$.

In the 10 central glands of the upper eye lid, the number of plugged glands decreased from $6.2 \%$ to $3.2 \%(-46 \%$ $P<0.0001)$; the proportion of eyes with a clear meibum more than doubled (from $7.5 \%$ to $16 \%$ ), the proportion of eyes with a solid meibum more than halved (from $33 \%$ to $14 \%$ ), and the average quality of the secretion changed from 1.7 (close to granular) to 1.3 (closer to cloudy) $(-24 \%, P<0.001)$; the number of glands that could not be expressed decreased from 4.7 to $2.8(-39 \%, P<0.0001)$; the proportion of eyes for which expressibility (the pressure required for expression) was heavy decreased from $30 \%$ to $22 \%$, and the proportion 
for which expressibility was minimal or mild increased from $9 \%$ to $20 \%$, while the average change modestly declined by $10 \%(P<0.01)$.

In the central two-thirds of the lower eyelid, the proportion of eyes with no gland dropout doubled (from 12\% to $25 \%$ ) and the proportion of eyes with $>66 \%$ dropout decreased from $7.5 \%$ to $1.2 \%$. On average, this component decreased by $35 \%(P<0.0001)$.

An unexpected result was the number of nonatrophied glands, which is not a part of the MGS score. Interestingly, this number increased after treatment with Optima ${ }^{\mathrm{TM}}$ IPL + MGX: on average, the number of nonatrophied glands increased by 2.5 glands, from $17.2 \pm 1.1$ at $B L$ to $19.8 \pm 1.3$ at FU3 $(P<0.0001)$. This result is intriguing, as the resuscitation of atrophied glands is highly unlikely - if not impossible. It is possible that some of the glands that were considered atrophied at BL were not completely atrophic but recovered at FU3.

\section{CFS}

CFS was evaluated using the Baylor grading scheme.

In site 1 , the average CFS decreased from BL by $-57 \%,-43 \%$, and $-58 \%$ at the FU1, FU2, and FU3, respectively (Table 2). The proportion of eyes with CFS scores below the cutoff (CFS < 10) increased from $63 \%$ at BL to $95 \%$ at the FU3 (Table 3). The proportion of eyes with CFS $=0$ (no dots or areas of confluence) increased from $0 \%$ at BL to $24 \%$ at the FU3. With respect to OR, a normal CSF score was 10 times more likely to be observed at the FU3 than at the BL (Figure 3).

In site 2, corneal abrasions or scratches were rarely observed by the investigator and most of the CFS values reported by the investigator were null. Hence, analysis of CFS was omitted for this site. It is possible that the staining method used in site 2 impaired this investigator's ability to detect corneal defects.

\section{TFO}

An eye was considered as abnormal with respect to tear osmolarity if at least one of the following conditions held: if $\mathrm{TFO}$ was $\geq 310 \mathrm{mOsm} / \mathrm{L}$, or if $\Delta \mathrm{TFO}$, the difference between this eye and the fellow eye, was $\geq 8 \mathrm{mOsm} / \mathrm{L}$. At BL, 51 eyes (64\%) satisfied this requirement. This number declined to $37(46 \%), 37(46 \%)$, and $34(43 \%)$ at the FU1, FU2, and FU3, respectively. Next, we examined how Optima $^{\text {TM }}$ IPL combined with MGX affected each of these two conditions separately.
For the analysis of TFO, we considered only 20 eyes for which the BL TFO was $\geq 310 \mathrm{mOsm} / \mathrm{L}$. In the other 60 eyes, TFO was normal and therefore - with respect to their TFO - these eyes were not candidates for improvement. In the 20 eyes included in the analysis, the mean TFO decreased from $322 \pm 19$ to $298 \pm 12 \mathrm{mOsm} / \mathrm{L}$ at the FU1. Similar means were obtained at the second and third follow-ups as well (Table 2). While a 7\% reduction in TFO may seem modest, according to Versura et al, ${ }^{25}$ a change from 322 to $298 \mathrm{mOsm} / \mathrm{L}$, a $7.5 \%$ reduction, corresponds to a change from severe to mild DED. Moreover, TFO decreased below the cutoff value in 17 (85\%) of the 20 eyes (Table 3). By definition, all eyes taken for this analysis had abnormal TFO at BL. Hence, ORs could not be calculated.

For the analysis of $\triangle \mathrm{TFO}$, only patients for whom the $\Delta \mathrm{TFO}$ was $\geq 8 \mathrm{mOsm} / \mathrm{L}$ were examined. Again, patients with a smaller BL $\triangle \mathrm{TFO}$ were excluded from this analysis, as by definition these patients were not candidates for improvement. Since all patients satisfying the requirement for analysis had abnormal $\triangle \mathrm{TFO}$ at BL, ORs were undefined. For patients included in this analysis, on average, $\triangle \mathrm{TFO}$ decreased from $17.8 \mathrm{mOsm} / \mathrm{L}$ at BL to $6.3 \mathrm{mOsm} / \mathrm{L}$ at the FU3 (Table 2). Of the 24 patients who satisfied $\Delta \mathrm{TFO} \geq 8 \mathrm{mOsm} / \mathrm{L}$ at the BL, $16(67 \%)$ patients presented to the FU3 with a normal $\Delta$ TFO (Table 3 ).

\section{LLT}

LLT was measured with the interferometer LipiView at site 1 only. No change in LLT was observed (Tables 2 and 3 and Figure 3 ).

\section{Number of signs and symptoms}

To summarize these results, we examined the following question: of the five defining measures, ie, the measures used for inclusion of an eye in the study, how many switched from abnormal to normal as a result of treatment with Optima ${ }^{\mathrm{TM}}$ IPL followed by MGX? For TBUT, SPEED, MGS, and CFS, the measure was defined as abnormal if it was $\leq 10 \mathrm{~s}, \geq 10,>10$, and $\geq 10$, respectively. For TFO, the measure was considered abnormal if either $\mathrm{TFO} \geq 310 \mathrm{mOsm} / \mathrm{L}$ or $\Delta \mathrm{TFO}$ (the difference between its TFO and the TFO of the fellow eye) $\geq 8 \mathrm{mOsm} / \mathrm{L}$.

It is important to recall that the condition for inclusion in the study was that at least two of these measures were compatible with DED. In this study, the median number of signs/symptoms decreased from 3 at the BL to 1 at the FU3. On average, the number of signs/symptoms decreased 
from $3.3 \pm 0.1$ at the BL to $1.4 \pm 0.1$ at the FU3 (paired $t$-test, $P<0.001)$.

\section{Discussion}

In this prospective study, we evaluated the effect of Optima ${ }^{\mathrm{TM}}$ IPL combined with MGX on eyes affected with moderate to severe MGD. All five defining signs/symptoms responded positively to the treatment, both in terms of average values (numerical analysis) and in terms of the proportion of eyes with signs or symptoms consistent with DED (categorical analysis). The average patient improved from a moderate/ severe state to a mild state of DED. A subgroup analysis suggested that the treatment was equally effective for patients with moderate/severe rosacea and for patients with no or a mild form of rosacea.

It is widely accepted that a thicker lipid layer increases the stability of the tear film, thus better preventing the evaporation of the aqueous component. ${ }^{20}$ Our study, however, did not show any change in LLT, in contrast with clinically significant changes in TBUT and all other outcome measures. Why were not these improvements accompanied with a corresponding increase in LLT? Our result corroborates with other studies, which found no correlation between TBUT and LLT. ${ }^{27}$ The importance of LLT is, indeed, increasingly challenged. King-Smith et $\mathrm{al}^{28}$ found that a thicker lipid layer does not necessarily imply slower evaporation, if the lipid layer is deficient in composition and/or structure, as is indeed the case in MGD. ${ }^{29}$ It is plausible that in our study, improvements in TBUT and other outcome measures are related to qualitative changes in the composition or structure of the meibum rather than merely quantitative changes in its thickness.

Our results are in agreement with several trials, which have demonstrated the efficacy of IPL for the treatment of MGD. ${ }^{13-17}$ The mechanism of action is, however, not known. One possibility is that IPL acts by treating the cutaneous forms of rosacea, in patients clinically (or even subclinically) affected with this inflammatory disease. The beneficial effects of IPL on acne rosacea are well known and have been extensively documented: ${ }^{10,11,30-32}$ the IPL energy is absorbed in abnormal blood vessels and causes their destruction by thrombolysis. Abnormal blood vessels release chemokines, cytokines, and other proinflammatory agents. By destroying these blood vessels, a major source of inflammatory mediators is reduced. ${ }^{14}$ In addition, skin diseases such as rosacea are characterized by an increased epithelial turnover. Large scales can detach from the epidermal surface and may obstruct the meibomian glands. ${ }^{33}$ By treating rosacea, this obstruction can be considerably reduced. Other explanations include facilitating expression by softening the meibum as a result of heat transfer to the eyelids and meibomian glands, ${ }^{13}$ upregulating anti-inflammatory molecules, such as interleukins, ${ }^{34}$ augmenting the production of collagen by stimulating fibroblasts; $;{ }^{35}$ and eradicating Demodex mites, ${ }^{36}$ which thrive on rosacea skin and are infested with Bacillus olerinus. ${ }^{37}$ This would have the indirect effect on decreasing the bacterial load on the eyelids.

Whatever the mechanism of action, this study and others support the notion that IPL is efficacious in treating MGD and DED.

\section{Limitations}

There were several limitations to this study. One limitation was that, in the two sites, different methods were used to stain the ocular surface. In site 1 , the ocular surface was stained by touching the conjunctiva with a fluorescein sodium strip, and in site 2 , the ocular surface was stained by instilling one drop of Fluess solution. As a result, the TBUT values measured in site 2 were considerably elevated in comparison with the TBUT values measured in site 1 . CSF data were affected as well. Consequently, the TBUT and CFS data from the two sites could not be pooled, and the TBUT and CSF analyses had to be conducted separately for each site.

Another limitation of this study was the design. Because the trial was single arm and not randomized controlled, changes observed during the study could be attributed to placebo or Hawthorne effects (the latter, being the process by which a subject is aware of being followed and observed and, as a result, changes his/her routine behavior or hygiene habits, thereby affecting the clinical outcome). Another confounder is that patients generally seek solutions when their symptoms become difficult to tolerate. Hence, it is possible that participants in this study enrolled when their symptoms were at their very worst. If so, improvement during the study is expected - not necessarily because of treatment, but simply due to regression to the mean.

The third limitation was that the treatment included Optima $^{\mathrm{TM}}$ IPL sessions immediately followed by MGX, as was done in other studies. ${ }^{13,15,16}$ Although expression does not address the root cause, it may help reduce the severity of the condition, by clearing clogged meibomian glands and allowing them to heal and function more properly. It is, therefore, unclear whether the observed improvements in signs and symptoms of DED in our study result from the Optima ${ }^{\mathrm{TM}}$ IPL itself, from the expression of meibomian glands, or from a combination of both. 
Finally, the fourth limitation was that among the five inclusion criteria, of which two were required for inclusion, only one was specific to MGD (the MGS). This means that a small proportion of eyes included in the study could have signs or symptoms of dry eye but not necessarily due to MGD. Retrospective exclusion of these eyes, however, did not have a significant effect on the results.

Furthermore, randomized controlled studies are required to address these limitations and shed light on these questions and, in particular, the clean effect of monotherapy IPL for relieving the signs and symptoms of MGD.

\section{Conclusion}

In subjects with moderate to severe MGD, IPL combined with MGX reduced the number and severity of symptoms and signs of DED. Except for LLT, all examined outcome measures significantly improved after 15 weeks. These results support the efficacy of IPL + MGX in relieving both signs and symptoms of DED secondary to MGD.

\section{Disclosure}

SJD, RNG, SCB, and DNC are consultants to Lumenis. The authors report no other conflicts of interest in this work.

\section{References}

1. Lemp MA, Crews LA, Bron AJ, Foulks GN, Sullivan BD. Distribution of aqueous-deficient and evaporative dry eye in a clinic-based patient cohort. Cornea. 2012;31(5):472-478.

2. Ding J, Sullivan DA. Aging and dry eye disease. Exp Gerontol. 2012;47(7):483-490.

3. Baudouin C, Messmer EM, Aragona P, et al. Revisiting the vicious circle of dry eye disease: a focus on the pathophysiology of meibomian gland dysfunction. Br J Ophthalmol. 2016;100(3):300-306.

4. Qiao J, Yan X. Emerging treatment options for meibomian gland dysfunction. Clin Ophthalmol. 2013;7:1797-1803.

5. Akpek EK, Merchant A, Pinar V, Foster CS. Ocular rosacea: patient characteristics and follow up. Ophthalmology. 1997;104(11):1863-1867.

6. Quarterman MJ, Johnson DW, Abele DC, Lesher JL Jr, Hull DS, Davis LS. Ocular rosacea. Signs, symptoms, and tear studies before and after treatment with doxycycline. Arch Dermatol. 1997;133(1):49-54.

7. Ghanem VC, Mehra N, Wong S, Mannis MJ. The prevalence of ocular signs in acne rosacea: comparing patients from ophthalmology and dermatology clinics. Cornea. 2003;22(3):230-233.

8. Patiño-Rodríguez B, Rodríguez-García A, Díaz J, Perfecto-Avalos Y. External ocular surface changes in ocular rosacea patients. Revista Mexicana de Oftalmologia. 2012;86(2):86-96.

9. Viso E, Rodríguez-Ares MT, Abelenda D, Oubiña B, Gude F. Prevalence of asymptomatic and symptomatic meibomian gland dysfunction in the general population of Spain. Invest Ophthalmol Vis Sci. 2012; 53(6):2601-2606.

10. Papageorgiou P, Clayton W, Norwood S, Chopra S, Rustin M. Treatment of rosacea with intense pulsed light: significant improvement and long-lasting results. Br J Dermatol. 2008;159(3):628-632.

11. Mark KA, Sparacio RM, Voigt A, Marenus K, Sarnoff DS. Objective and quantitative improvement of rosacea-associated erythema after intense pulsed light treatment. Dermatol Surg. 2003;29(6):600-604.
12. Toyos R, Buffa CM, Youngerman S. Case report: Dry-eye symptoms improve with intense pulsed light treatment. Eye World News Magazine. 2005 September.

13. Toyos R, McGill W, Briscoe D. Intense pulsed light treatment for dry eye disease due to meibomian gland dysfunction: a 3-year retrospective study. Photomed Laser Surg. 2015;33(1):41-46.

14. Craig JP, Chen YH, Turnbull PR. Prospective trial of intense pulsed Light for the treatment of meibomian gland dysfunction. Invest Ophthalmol Vis Sci. 2015;56(3):1965-1970.

15. Vegunta S, Patel D, Shen JF. Combination therapy of intense pulsed light therapy and meibomian gland expression (IPL/MGX) can improve dry eye symptoms and meibomian gland function in patients with refractory dry eye: a retrospective analysis. Cornea. 2016;35(3): 318-322.

16. Gupta PK, Vora GK, Matossian C, Kim M, Stinnett S. Outcomes of intense pulsed light therapy for treatment of evaporative dry eye disease. Can J Ophthalmol. 2016;51(4):249-253.

17. Jiang X, Lv H, Song H, et al. Evaluation of the safety and effectiveness of intense pulsed light in the treatment of meibomian gland dysfunction. J Ophthalmol. 2016;2016:1910694.

18. Norn MS. Desiccation of the precorneal film. I. Corneal wetting-time. Acta Ophthalmol (Copenh). 1969;47(4):865-880.

19. Ngo W, Situ P, Keir N, Korb D, Blackie C, Simpson T. Psychometric properties and validation of the standard patient evaluation of eye dryness questionnaire. Cornea. 2013;32(9):1204-1210.

20. Blackie CA, Solomon JD, Scaffidi RD, Greiner JV, Lemp MA, Korb DR. The relationship between dry eye symptoms and lipid layer thickness. Cornea. 2009;28(7):789-794.

21. Foulks GN, Bron AJ. Meibomian gland dysfunction: a clinical scheme for description, diagnosis, classification, and grading. Ocul Surf. 2003; 1(3):107-126.

22. Rao K, Farley WJ, Pflugfelder SC. Association between high tear epidermal growth factor levels and corneal subepithelial fibrosis in dry eye conditions. Invest Ophthalmol Vis Sci. 2010;51(2):844-849.

23. Fenner BJ, Tong L. Corneal staining characteristics in limited zones compared with whole cornea documentation for the detection of dry eye subtypes. Invest Ophthalmol Vis Sci. 2013;54(13):8013-8019.

24. Lemp MA, Bron AJ, Baudouin C, et al. Tear osmolarity in the diagnosis and management of dry eye disease. Am J Ophthalmol. 2011;151(5): 792.e1-798.e1.

25. Versura P, Profazio V, Campos EC. Performance of tear osmolarity compared to previous diagnostic tests for dry eye diseases. Curr Eye Res. 2010;35(7):553-564.

26. Finis D, Pischel N, Schrader S, Geerling G. Evaluation of lipid layer thickness measurement of the tear film as a diagnostic tool for Meibomian gland dysfunction. Cornea. 2013;32(12): 1549-1553.

27. Fenner BJ, Tong L. More to stable tears than thickness of the tear film lipid layer. Invest Ophthalmol Vis Sci. 2015;56(3):1601.

28. King-Smith PE, Reuter KS, Braun RJ, Nichols JJ, Nichols KK. Tear film breakup and structure studied by simultaneous video recording of fluorescence and tear film lipid layer images. Invest Ophthalmol Vis Sci. 2013;54(7):4900-4909.

29. Butovich IA, Lu H, McMahon A, et al. Biophysical and morphological evaluation of human normal and dry eye meibum using hot stage polarized light microscopy. Invest Ophthalmol Vis Sci. 2014; 55(1):87-101.

30. Taub AF. Treatment of rosacea with intense pulsed light. $J$ Drugs Dermatol. 2003;2(3):254-259.

31. Angermeier MC. Treatment of facial vascular lesions with intense pulsed light. J Cutan Laser Ther. 1999;1(2):95-100.

32. Liu J, Liu J, Ren Y, Li B, Lu S. Comparative efficacy of intense pulsed light for different erythema associated with rosacea. $J$ Cosmet Laser Ther. 2014;16(6):324-327.

33. Henriquez AS, Korb DR. Meibomian glands and contact lens wear. Br J Ophthalmol. 1981;65(2):108-111. 
34. Byun JY, Choi HY, Myung KB, Choi YW. Expression of IL-10, TGF- $\beta 1$ and TNF- $\alpha$ in cultured keratinocytes (HaCaT Cells) after IPL treatment or ALA-IPL photodynamic treatment. Ann Dermatol. 2009;21(1): $12-17$.

35. Cuerda-Galindo E, Díaz-Gil G, Palomar-Gallego MA, LinaresGarcíaValdecasas R. Increased fibroblast proliferation and activity after applying intense pulsed light 800-1200 nm. Ann Anat. 2015; 198:66-72.
36. Prieto VG, Sadick NS, Lloreta J, Nicholson J, Shea CR. Effects of intense pulsed light on sun-damaged human skin, routine, and ultrastructural analysis. Lasers Surg Med. 2002;30(2):82-85.

37. Szkaradkiewicz A, Chudzicka-Strugała I, Karpiński TM, et al. Bacillus oleronius and Demodex mite infestation in patients with chronic blepharitis. Clin Microbiol Infect. 2012;18(10):1020-1025.

\section{Publish your work in this journal}

Clinical Ophthalmology is an international, peer-reviewed journal covering all subspecialties within ophthalmology. Key topics include: Optometry; Visual science; Pharmacology and drug therapy in eye diseases; Basic Sciences; Primary and Secondary eye care; Patient Safety and Quality of Care Improvements. This journal is indexed on

Submit your manuscript here: http://www.dovepress.com/clinical-ophthalmology-journal

\section{Dovepress}

PubMed Central and CAS, and is the official journal of The Society of Clinical Ophthalmology (SCO). The manuscript management system is completely online and includes a very quick and fair peer-review system, which is all easy to use. Visit http://www.dovepress.com/ testimonials.php to read real quotes from published authors. 